\title{
Broken symmetries and directed collective energy transport in spatially extended systems
}

Flach, S.; Zolotaryuk, Yaroslav; Miroshnichenko, A. E.; Fistul, M. V.

Published in:

Physical Review Letters

Link to article, DOI:

10.1103/PhysRevLett.88.184101

Publication date:

2002

Document Version

Publisher's PDF, also known as Version of record

Link back to DTU Orbit

Citation (APA):

Flach, S., Zolotaryuk, Y., Miroshnichenko, A. E., \& Fistul, M. V. (2002). Broken symmetries and directed collective energy transport in spatially extended systems. Physical Review Letters, 88(18), 184101. https://doi.org/10.1103/PhysRevLett.88.184101

\section{General rights}

Copyright and moral rights for the publications made accessible in the public portal are retained by the authors and/or other copyright owners and it is a condition of accessing publications that users recognise and abide by the legal requirements associated with these rights.

- Users may download and print one copy of any publication from the public portal for the purpose of private study or research.

- You may not further distribute the material or use it for any profit-making activity or commercial gain

- You may freely distribute the URL identifying the publication in the public portal 


\title{
Broken Symmetries and Directed Collective Energy Transport in Spatially Extended Systems
}

\author{
S. Flach, ${ }^{1}$ Y. Zolotaryuk, ${ }^{2}$ A. E. Miroshnichenko, ${ }^{1}$ and M. V. Fistul ${ }^{1}$ \\ ${ }^{1}$ Max-Planck-Institut für Physik komplexer Systeme, \\ Nöthnitzer Strasse 38, D-01187 Dresden, Germany \\ ${ }^{2}$ Section for Mathematical Physics, IMM, Technical University of Denmark, \\ DK-2800, Lyngby, Denmark \\ (Received 8 October 2001; published 17 April 2002)
}

\begin{abstract}
We study the appearance of directed energy current in homogeneous spatially extended systems coupled to a heat bath in the presence of an external ac field $E(t)$. The systems are described by nonlinear field equations. By making use of a symmetry analysis, we predict the right choice of $E(t)$ and obtain directed energy transport for systems with a nonzero topological charge $Q$. We demonstrate that the symmetry properties of motion of topological solitons (kinks and antikinks) are equivalent to the ones for the energy current. Numerical simulations confirm the predictions of the symmetry analysis and, moreover, show that the directed energy current drastically increases as the dissipation parameter $\alpha$ reduces.
\end{abstract}

DOI: $10.1103 /$ PhysRevLett.88.184101

PACS numbers: 05.45.Yv, 05.60.Cd

The idea of rectifying energy transport with the help of fluctuations has been discussed for several years in connection with molecular motors and other nonequilibrium properties of biological systems [1], electrical currents in superlattices [2], and voltages in Josephson junction coupled systems $[3,4]$, to name a few. The fluctuations have zero mean value; i.e., the dc component is absent. The reduction of the underlying problem, namely, directed energy transport, to a particle moving in a space-periodic but asymmetric (ratchet) potential allowed one to study the resulting directed current in great detail [5] (for a recent review see [6]). A recently elaborated symmetry approach to this problem established a clear relationship between directed currents and broken space-time symmetries [7-9]. The essential step was to separate the unavoidable correlations in the fluctuations from the uncorrelated ones. This is easily obtained by replacing the fluctuations as a superposition of ac driving fields and uncorrelated white noise. An important consequence is that the symmetries may be broken either by violating the reflection symmetry of the potential in space or by violating the shift symmetry of the ac fields. Thus, a particle may display a directed motion also in the case of a space-symmetric potential. Another interesting result is the persistence of directed currents in the Hamiltonian limit of systems exposed to ac fields but decoupled from the heat bath $[7,8]$.

A very important question is whether the symmetry approach can be generalized to the case of interacting manyparticle systems (for related studies see [6,10-12]). In this Letter, we focus on nonlinear partial differential equations, which can be considered as classical analogues of quantum models of interacting particles [13]. We show that such systems allow for a directed energy transport when being driven by a proper combination of ac forces. The success of such a generalization will not only underline the general validity of the symmetry analysis, but will also allow for a systematic symmetry analysis of nonadiabatic nonlinear response functionals for many-body theories.
We study the properties of nonlinear Klein-Gordon equations for a scalar field $\varphi$ which depends both on a spatial coordinate $x$ and time $t$ :

$$
\begin{gathered}
\varphi_{, t t}-c_{0}^{2} \varphi_{, x x}+\alpha \varphi_{, t}+\frac{d U}{d \varphi}=f(t, x), \\
f(t, x)=E(t)+\xi(t, x),
\end{gathered}
$$

where $c_{0}$ is the limiting propagation speed of small amplitude plane waves, $\alpha$ is the dissipation parameter determining the inverse relaxation time in the system, and $f_{z z} \equiv$ $\partial f / \partial z$. The ac field $E(t)$ has zero mean and period $T$. The Gaussian white noise $\xi$ is characterized by the standard correlation function $\left\langle\xi(t, x) \xi\left(t^{\prime}, x^{\prime}\right)\right\rangle=2 \frac{\alpha}{\beta} \delta(x-$ $\left.x^{\prime}\right) \delta\left(t-t^{\prime}\right)$, where $\beta$ is the inverse temperature. The potential $U(z)$ is assumed to have several minima of identical height for $z=z_{i}, i=1,2, \ldots$. We choose fixed boundary conditions: $\varphi(x \rightarrow-\infty)=z_{l}, \varphi(x \rightarrow+\infty)=z_{m}$. With these boundary conditions, we define the topological charge $Q=m-l$ which will be of importance in the following. The energy current density $j$, generated by the field $\varphi$ in the absence of external forcing and damping, can be obtained from the standard continuity equation [14]. It leads to the following expression for the energy current $J$ :

$$
J(t)=\int_{-\infty}^{+\infty} j d x=-c_{0}^{2} \int_{-\infty}^{+\infty} \varphi_{, t} \varphi_{, x} d x
$$

Next we turn to the symmetry analysis of (1) and (2). It is carried out in the absence of $\xi$ in (1), as it does not affect the symmetries. We note that, for $Q=0, J$ changes its sign under the transformation $x \rightarrow-x$. Equation (1) is invariant under the space inversion. Consequently, we conclude that, for zero topological charge, $Q=0$, the time-averaged energy current $\langle J\rangle(\tau)=\frac{1}{\tau} \int_{0}^{\tau} J(t) d t$ vanishes exactly for large averaging times $\tau$.

In order to study the possibility of the appearance of a nonzero energy current in the presence of a nonzero topological charge, $Q \neq 0$, we note that the topological charge 
$Q \neq 0$ is invariant under the combined symmetry operation $x \rightarrow-x$ and $\varphi \rightarrow-\varphi+z_{l}+z_{m}$. The same symmetry operation changes the sign of the energy current, and all we need is to ensure that the equation (1) remains invariant. This is now possible only if the ac field possesses shift symmetry and the potential $U(z)$ is symmetric around $z=\left(z_{l}+z_{m}\right) / 2$. Thus, we arrive at the symmetry operation,

$$
x \rightarrow-x, \quad \varphi \rightarrow-\varphi+z_{l}+z_{m}, \quad t \rightarrow t+\frac{T}{2},
$$

which leaves the equation (1) invariant and changes the sign of the energy current as the constraints,

$$
\begin{gathered}
E(t)=-E(t+T / 2), \\
U\left(z-\frac{z_{l}+z_{m}}{2}\right)=U\left(-z+\frac{z_{l}+z_{m}}{2}\right),
\end{gathered}
$$

hold. In such a case, we may conclude that the directed energy current vanishes [15]. Violating (4) we lose the symmetry (3) and, consequently, may expect a nonzero energy current provided no further hidden symmetries are overlooked.

Most interesting is that the conditions (4) can be violated by choosing a function $E(t)$ which is not shift symmetric. A simple choice is

$$
E(t)=E_{1} \cos \omega t+E_{2} \cos (2 \omega t+\Delta),
$$

with $E_{1} \neq 0$ and $E_{2} \neq 0$. In particular, such an ac field allows one to generate a nonzero energy current in the well-known sine-Gordon equation with

$$
U(z)=-\cos z
$$

which we will consider in the following. At the same time, we stress here that any other choice of the ac drive $E(t)$ which is not shift symmetric (e.g., a corresponding sequence of pulses) will do the job, as well as any other potential $U(z)$ which meets the above described conditions.

Of special interest is that, in the Hamiltonian limit as $\alpha \rightarrow 0$, time reversal symmetry is recovered: The symmetry operation $t \rightarrow-t$ leaves (1) invariant, provided $E(t)$ is symmetric: $E(t)=E(-t)$. As time reversal always changes the sign of the energy current (2), zero average energy current will be a consequence. Thus, for the underdamped case $\alpha \ll 1$, we expect a change of sign of the energy current upon varying $\Delta$ because, for the particular values of $\Delta=0, \pi$ time reversal is approximately restored and the directed energy current disappears.

As discussed above, a necessary condition for a directed energy current is a nonzero topological charge $Q$. It implies the presence of an excess of kinks over antikinks or vice versa. The simplest case is $Q=1$ as a single unpinned kink is present on average in the system. As a kink possesses a nonzero rest energy $E_{k}=8 c_{0}$, it is natural to expect that the nonzero energy current is generated by a directed motion of such a kink. Indeed, assuming that the kink position $X$ and velocity $V$ are described by expressions

$$
\begin{gathered}
X(t)=\frac{1}{2 \pi Q} \int_{-\infty}^{+\infty} x \varphi_{, x} d x, \\
V=\frac{1}{2 \pi Q} \int_{-\infty}^{+\infty} x \varphi_{, x t} d x,
\end{gathered}
$$

we observe that the symmetry operation (3) changes the sign of $V$. Thus, we find that, if the energy current vanishes by symmetry, the same is true for the average velocity of a kink. This shows the intimate connection between a directed energy current and coherent kink excitations as carriers of such an energy. In [12] a directed kink motion was obtained numerically for the first time for the case (5),(6) together with a study of its complex dynamics. Note that in [16-18] a directed kink transport has been obtained by breaking the reflection symmetry of $U(z)$ for $E_{2}=0$.

If the temperature and thus the amplitude of $\xi$ is large enough, additional kink-antikink pairs are excited in the course of evolution. Since the space inversion $(x \rightarrow-x)$ does not change Eq. (1), transforms $Q \rightarrow-Q$, and kinks into antikinks with inverted velocities, the average velocity of a kink-antikink pair is zero. Consequently, the generation of kink-antikink pairs does not affect the net energy current of the system predefined by the value of topological charge $Q$.

With these analytical results we turn to a numerical investigation. We used a standard discretization scheme and integrated the following coupled differential equations using a fourth-order Runge-Kutta method (see also [19]):

$$
\begin{aligned}
\ddot{\phi}_{n}-c_{0}^{2}\left(\phi_{n-1}-2 \phi_{n}+\phi_{n+1}\right)+ & \\
\alpha \dot{\phi}_{n}+\sin \phi_{n} & =f_{n}(t) .
\end{aligned}
$$

Here $n$ is an integer representing the discretization in space. We choose the following parameters: $\beta=100$, $c_{0}^{2}=10, E_{1}=0.2, E_{2}=0.2, \omega=0.1, \tau=50000,1 \leq$ $n \leq N=420$, and $Q=1$. The boundary condition was implemented using $\phi_{N+1}=\phi_{1}+2 \pi$ with the initial condition of a single kink solution [20]. The particular value of $c_{0}$ ensures that the effects of discreteness on the kink motion may be neglected [21].

The time-averaged energy current $\langle J\rangle$ as a function of the phase shift $\Delta$ for three different values of dissipation parameter $\alpha=0.01,0.05$, and 0.2 is shown in Fig. 1. We obtain a strong dependence of the directed energy current $\langle J\rangle$ on $\Delta$ with sign changes, as expected. Furthermore, the maximum values of the energy current increase substantially as the dissipation parameter $\alpha$ reduces, similar to the case of a single particle moving in a space-periodic potential [8]. Especially, we find an increase by a factor of 50 when the damping $\alpha$ decreases from 0.2 to 0.01 . Finally, we clearly observe that, as the parameter $\alpha$ reduces, the zeros of the curves tend to the positions determined by time reversal symmetry, $\Delta=0, \pi$. We computed in a 


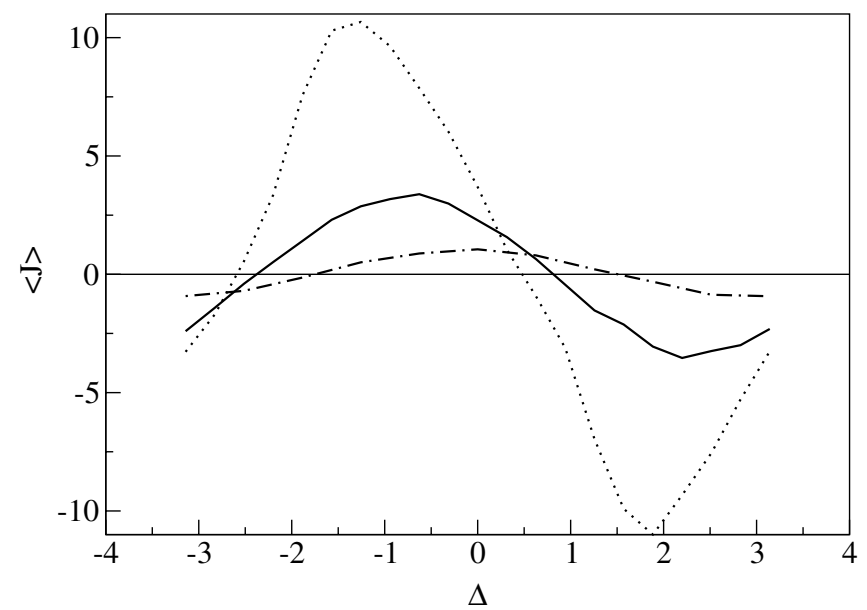

FIG. 1. Dependence of the time-averaged energy current $\langle J\rangle$ on the phase shift $\Delta$. Solid line, $\alpha=0.05$; dotted line, $\alpha=$ 0.01 ; dash-dotted line, $\alpha=0.2$. Note that the latter case $\alpha=$ 0.2 is scaled by a factor of 5 , so the real values of $\langle J\rangle$ are 5 times less than they appear in the plot.

similar way the time averages of $V$ as defined in (7), and obtained identical results upon variation of $\Delta$ and $\alpha$. It shows that indeed kink motion is responsible for the energy transfer.

In these simulations, the intensity of the white noise $\xi$ was small enough to prevent an excitation of a kinkantikink pair during the simulation time. Nevertheless a very long simulation should also show up with a spontaneous creation of such pairs. In order to see that this event does not influence the symmetry analysis and especially does not change the obtained values of the energy current (cf. above considerations), we proceed with a comparison. In Fig. 2 we plot the dependence of the time-averaged energy current $\langle J\rangle(\tau)$ on the time of averaging $\tau$ for $\alpha=$ 0.01 and $\Delta=\pi / 2$, as a single kink is present in the system. In the same figure, we plot the result for the case of additionally exciting a kink-antikink pair [20]. We indeed observe that the value for the time-averaged energy current does not depend on the number of additional kink-antikink pairs. As the simulation time increases even further, soliton collisions eventually may lead to the dissipation of the pair, with one single kink remaining in the system.

Let us discuss possible mechanisms of energy current rectification in spatially homogeneous extended systems through directed kink motion. First we notice that in many cases the kink motion may be mapped to the classical mechanics of a macroscopic particle [22]. However, a free particle that is subject to a spatially homogeneous ac force does not display a directed motion at all. One possible source of the observed directed current can be relativistic effects which are inherent to the considered nonlinear Klein-Gordon field equations. They can be explicitly obtained in some cases using projection techniques to derive effective equations of motion for the kink center [22]. In such a regime the kink motion is mapped onto the one

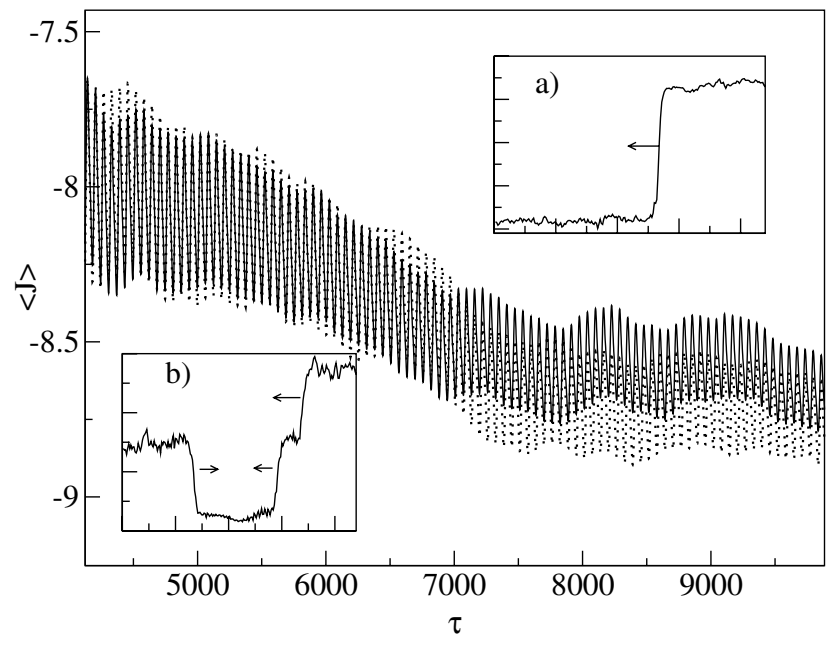

FIG. 2. Dependence of the time-averaged energy current $\langle J\rangle$ on the averaging time $\tau$ for $\Delta=\pi / 2$ and $\alpha=0.01$ for the two cases: a single kink in the system (solid line), a single kink and an additional kink-antikink pair in the system (dotted line). The insets (a) and (b) show snapshots of the field $\varphi$ as a function of $x$ at some time during the simulation for these two cases. Arrows indicate the direction of time-averaged motion of the $\operatorname{kink}(\mathrm{s})$ and the antikink.

of a relativistic particle in the presence of damping and the ac field $E(t)$. The dependence of the velocities on $\alpha$ and $\omega[12]$ is, however, in sharp contrast to our findings. Thus we argue that the origin of the observed strong rectification in the underdamped limit is due to the nonadiabatic excitation of internal kink modes and their interaction with the translational kink motion $[12,17,23]$. That will be discussed in detail in a future work.

It is worthwhile to mention a number of other ways to generate a nonzero energy current. One case is to consider strongly discrete systems where the appearance of a nonnegligible Peierls-Nabarro potential can generate a net kink motion through the lattice. Also all sorts of perturbations in the form of spatial modulations may act similar to discretization effects [4,24]. Yet another way of breaking the symmetry has been analyzed in [25] where in addition to an ac force $E(t)=\cos t$ the potential $U(z, t)=$ $\left(z^{2}-1\right)^{2}+z^{2} \sin t$ was assumed to be time dependent. The application of our symmetry analysis yields that the symmetries (3) are violated and directed energy transport occurs. It is also interesting to recall that a number of publications have been dealing with directed kink motion in asymmetric double well potentials $[18,26]$. The asymmetry was chosen in a way to entropically favor one equilibrium over the other one (still keeping the energy values of the equilibria identical). Even Gaussian white noise leads then to the directed motion of a kink. However, additional ac fields have been shown to counterbalance the entropic force.

While it is certain that our results may be applied to many different physical situations, where the sine-Gordon equation is replaced by other Klein-Gordon equations with 
topological excitations, we stress that a particular system, namely, an annular long Josephson junction with a trapped fluxon, is perhaps the ideal case to verify our results. This system is described precisely by (1) $[23,27,28]$. While in [23] experimental results for $E_{2}=0$ have been presented, all one needs is to add the second harmonics $E_{2} \neq 0$ and vary the phase shift $\Delta$. The analogue of the ac field is here an ac homogeneous microwave radiation which traverses the junction. Under these conditions, a trapped fluxon displays a directed motion, and, correspondingly, a dc voltage drop across the junction appears. This effect is the analogue of the energy current discussed here. Notice here that the dc voltage drop oscillates with the phase shift $\Delta$ and substantially increases in the "strong" underdamped limit, $\alpha \ll \omega$.

We conclude this work with stating that the use of symmetries allowed to obtain a relationship between a directed energy current and directed kink motion. This may be of further interest as it shows an intimate connection between symmetry breakings and the relevance of coherent excitations and their properties in many-body theories. Finally, we note that nonlinear Klein-Gordon systems which do not admit kink or antikink excitations (zero topological charge) do not allow for directed energy currents induced by ac forces, and that the above considerations can be used without change for spatially discrete systems.

We thank M. Salerno and A.V. Ustinov for useful discussions. This work was supported by the Deutsche Forschungsgemeinschaft FL200/8-1 and the European Union through LOC-NET HPRN-CT-1999-00163.

[1] F. Jülicher, A. Ajdari, and J. Prost, Rev. Mod. Phys. 69, 1269 (1997).

[2] K. Seeger and W. Maurer, Solid State Commun. 27, 603 (1978); I. Goychuk and P. Hänggi, Europhys. Lett. 43, 503 (1998); K. N. Alekseev et al., Phys. Rev. Lett. 80, 2669 (1998); K. N. Alekseev and F. V. Kusmartsev, cond-mat/ 0012348.

[3] I. Zapata, J. Łuczka, F. Sols, and P. Hänggi, Phys. Rev. Lett. 80, 829 (1998); S. Weiss, D. Koelle, J. Müller, R. Gross, and K. Barthel, Europhys. Lett. 51, 499 (2000); E. Trias, J. J. Mazo, F. Falo, and T. P. Orlando, Phys. Rev. E 61, 2257 (2000).

[4] G. Carapella and G. Costabile, Phys. Rev. Lett. 87, 077002 (2001).

[5] R. Bartussek, P. Hänggi, and J. G. Kissner, Europhys. Lett. 28, 459 (1994).
[6] P. Reimann, Phys. Rep. 361, 57 (2002).

[7] S. Flach, O. Yevtushenko, and Y. Zolotaryuk, Phys. Rev. Lett. 84, 2358 (2000).

[8] O. Yevtushenko, S. Flach, Y. Zolotaryuk, and A. A. Ovchinnikov, Europhys. Lett. 54, 141 (2001).

[9] P. Reimann, Phys. Rev. Lett. 86, 4992 (2001).

[10] S. Cilla, F. Falo, and L. M. Floria, Phys. Rev. E 63, 031110 (2001).

[11] Z. Zheng, G. Hu, and B. Hu, Phys. Rev. Lett. 86, 2273 (2001).

[12] M. Salerno and Y. Zolotaryuk, Phys. Rev. E (to be published).

[13] R. Rajaraman, Solitons and Instantons (Elsevier, Amsterdam, 1987).

[14] T. Prosen and D. K. Campbell, Phys. Rev. Lett. 84, 2857 (2000).

[15] Notice here that even if the conditions (4) are satisfied a nonzero energy current may persist for a rather large finite time [see, for example, B. A. Malomed and A. V. Ustinov, Phys. Rev. B 64, 020302 (2001)]. However, in this case the energy current of an opposite sign also exists, and a proper averaging over different realizations leads to zero total directed current. This is the main reason of introducing a nonzero temperature $[\xi(t)$ in Eq. (1)] in order to ensure this averaging.

[16] F. Marchesoni, Phys. Rev. Lett. 77, 2364 (1996).

[17] M. Salerno and N. R. Quintero, nlin.PS/0107011.

[18] A. V. Savin, G. P. Tsironis, and A. V. Zolotaryuk, Phys. Rev. E 56, 2457 (1997).

[19] R. F. Fox, I. R. Gatland, R. Roy, and G. Vemuri, Phys. Rev. A 38, 5938 (1988).

[20] A single kink and a kink-antikink pair were introduced in the system through the exact one- and three-soliton solution of the unperturbed sine-Gordon equation; see A.E. Miroshnichenko, S. V. Dmitriev, A. A. Vasiliev, and T. Shigenari, Nonlinearity 13, 837 (2000).

[21] S. Flach and C. R. Willis, Phys. Rev. E 47, 4447 (1993).

[22] D. W. McLaughlin and A. C. Scott, Phys. Rev. A 18, 1652 (1978); M. Salerno and A. C. Scott, Phys. Rev. B 26, 2474 (1982).

[23] M. V. Fistul, E. Goldobin, and A. V. Ustinov, Phys. Rev. B 64, 092501 (2001).

[24] E. Goldobin, A. Sterck, and D. Koelle, Phys. Rev. E 63, 031111 (2001).

[25] A. L. Sukstanskii and K. I. Primak, Phys. Rev. Lett. 75, 3029 (1995).

[26] G. Constantini and F. Marchesoni, Phys. Rev. Lett. 87, 114102 (2001).

[27] N. Grønbech-Jensen, P. S. Lomdahl, and M. R. Samuelsen, Phys. Rev. B 43, 12799 (1991).

[28] M. V. Fistul and A. V. Ustinov, Phys. Rev. B 63, 024508 (2000). 